\title{
Anomalous dimensions and phase transitions in superconductors
}

\author{
Flavio S. Nogueira* \\ Centre de Physique Théorique, Ecole Polytechnique, F-91128 Palaiseau Cedex, FRANCE
}

(Received October 25, 2018)

\begin{abstract}
The anomalous scaling in the Ginzburg-Landau model for the superconducting phase transition is studied. It is argued that the negative sign of the $\eta$ exponent is a consequence of a special singular behavior in momentum space. The negative sign of $\eta$ comes from the divergence of the critical correlation function at finite distances. This behavior implies the existence of a Lifshitz point in the phase diagram. The anomalous scaling of the vector potential is also discussed. It is shown that the anomalous dimension of the vector potential $\eta_{A}=4-d$ has important consequences for the critical dynamics in superconductors. The frequency-dependent conductivity is shown to obey the scaling $\sigma(\omega) \sim \xi^{z-2}$. The prediction $z \approx 3.7$ is obtained from existing Monte Carlo data.

Pacs: 74.20.-z, 05.10Cc, 11.25.Hf
\end{abstract}

Typeset using REVTEX

*address after september 1st 2000: Institut für Theoretische Physik, Freie Universität Berlin, Arnimallee 14, D-14195 Berlin 


\section{INTRODUCTION}

The superconducting phase transition has received considerable attention in recent years. All this interest is due in part to the experimentally larger critical region in the high- $T_{c}$ materials [1]3]. This larger critical region, however, does not correspond to the inverted 3D $\mathrm{XY}\left(\mathrm{IXY} \mathrm{Y}_{3}\right.$ for short) universality class [4]. Instead, the observed critical behavior belongs to the ordinary 3D XY ( $X Y_{3}$ for short) universality class, meaning that the phase transition is governed by the neutral non-trivial Wilson-Fisher fixed point. Concerning the charged transition (that is, the $I X Y_{3}$ behavior), there is some progress from the theoretical side. Unfortunatly, the corresponding critical region remains experimentally out of reach.

Concerning the $\mathrm{IXY}_{3}$ regime, interesting precise numerical results on the anomalous scaling dimensions have been obtained recently by Sudbø and collaborators [6 using a lattice version of the Ginzburg-Landau (GL) model. Their results give a strong support to the duality scenario [4,5,9,34] which underlies the $I X Y_{3}$ behavior. The aim ot this paper is to provide an analysis of the anomalous scaling dimensions from the point of view of field theory. An important issue to be understood is the sign of the order parameter field anomalous dimension, $\eta$. As argued in Ref. [23] a negative $\eta$, though fulfilling the inequality $\eta>2-d$, would spoil some important properties that must be verified in any legitimate continuum (scaling) limit. A fundamental property, the positivity of the spectral weight of the Källen-Lehmann (KL) spectral representation of the 2-point correlation function, is violated if $\eta<0$. Kiometzis and Schakel [25] pointed out also that unitarity is violated if $\eta<0$. In fact, violation of the unitarity is an immediate consequence of the violation of the positivity of the spectral weight. We should note, however, that most renormalization group (RG) calculations give in general $\eta$ in the range $-1<\eta<0$ 12, 16, 18, 19 in $d=3(\epsilon=1$ in the context of the $\epsilon$-expansion). The only exception is the case where a mass (Proca) term is added explicitly for the gauge field, where the inequality $\eta \geq 0$ is satisfied [20]. This last situation corresponds just to the case of the continuum dual model where the gauge symmetry is global [9,21,23]. Since in the RG calculations $\eta$ is only slightly negative we 
may wonder if such a negativeness is not just an artifact of the approximations used. The situation is, however, much more subtle. The recent numerical simulations in the lattice of Nguyen and Sudbø [7] gives $\eta=-0.18$ [24]. The results in Ref. [7] are non-perturbative, in contrast to most RG calculations. In the RG context we can cite the work of Bergerhoff et al. [16] and the $1 / N$ expansion [12], both non-perturbative and giving also $\eta<0$.

From a thermodynamical point of view, the anomalous dimension $\eta_{A}$ of the vector potential has more far reaching consequences. Indeed, it plays an important role in a critical regime where the magnetic fluctuations are not negligible, such as in the $I X Y_{3}$ regime. Gauge invariance allows an exact determination of $\eta_{A}$ in $2<d<4$ dimensions. Indeed, its value is given simply by $\eta_{A}=4-d$. One important consequence of this result is the scaling $\lambda \sim \xi[18,21,30,23]$ where $\lambda$ is the penetration depth and $\xi$ is the correlation length.

In this paper we will discuss some interesting new aspects of the superconducting transition. We will focus the issue of the anomalous dimensions of fields, for both the scalar field and the gauge field. Our analysis should be applicable to superconductors in the type II regime, where we expect a second-order (charged) phase transition [4,5]. In section II the negativeness of $\eta$ will be shown to be a consequence of the existence of two singularities in the scalar 2-point bare correlation function at the critical point $(\mathrm{CP})$. One singularity happens at $p=0$ while the other one happens at a nonzero momentum $p=p^{\prime}$. This second singularity is related to the existence of a first-order phase transition regime. This singularity at nonzero momentum is at the origin of the negative sign of the $\eta$ exponent. Indeed, $\eta$ is negative because the order parameter wave function renormalization $Z_{\phi}$ is greater than one and this happens only if the corresponding critical 2-point correlation function has a pole at nonzero momentum. We argue that this behavior implies the existence of a Lifshitz point induced by gauge field fluctuations.

Another point of view is to study the small fluctuations around the Halperin-LubenskyMa (HLM) mean field theory [12]. This is done in section III, where the Gaussian fluctuations are calculated in order to study the positivity properties of the propagators. It turns out that the propagators are positive definite and no pole at $p \neq 0$ is found at the CP. Indeed, 
in order to find out such a pole it is necessary to compute the non-Gaussian fluctuations. The analysis of the section III shows that the functional integral has a well defined Gaussian measure.

In section IV we discuss the physical consequences of the anomalous scaling dimension of the magnetic vector potential. After reviewing some known properties like the scaling $\lambda \sim \xi$ [18,21, 30,23, we analyse the consequences of the magnetic fluctuations for the frequency dependent conductivity, $\sigma(\omega)$. As argued by Fisher et al. [31], in the $X Y_{3}$ regime scales as $\sigma(\omega) \sim|t|^{\nu(d-2-z)}$ (for the sake of generality we wrote the scaling relation in dimension $2<d<4$, that is, a $X Y_{d}$ regime). However, if the magnetic fluctuations are included the anomalous dimension of the vector potential is no longer equal to zero. This implies the dimension independent scaling $\sigma(\omega) \sim|t|^{\nu(2-z)}$. We point out that the scaling $\nu^{\prime}=\nu$ implied by $\lambda \sim \xi$ ( $\nu^{\prime}$ is the penetration depth exponent) is also dimension independent, in contrast with the dimension dependent result of the $X Y_{d}$ regime, $\nu^{\prime}=\nu(d-2) / 2$. In section $\mathrm{V}$ we infer from the Monte Carlo data of Lidmar et al. that $z \approx 3.7$ in the $I X Y_{3}$ regime, which is a translation of one unity of the result obtained by these authors $(z \approx 2.7)$. This difference is due to the fact that the scaling $\sigma(\omega) \sim|t|^{\nu(d-2-z)}$ was assumed in their Monte Carlo simulation of the $I X Y_{3}$ regime. Finally, we discuss the relevance of these ideas to the Bose-glass transition [26,27] in the direction perpendicular to the columnar defects, where a transverse Meissner effect happens [27.

\section{PHASE TRANSITIONS AND THE ORDER PARAMETER ANOMALOUS DIMENSION}

In order to fix the ideas, let us consider first the case of a scalar $O(2)$ invariant field theory with bare Lagrangian

$$
L=\left|\partial_{\mu} \phi\right|^{2}+m^{2}|\phi|^{2}+\frac{u}{2}|\phi|^{4} .
$$

Such a theory has a non-trivial infrared stable fixed point at $d=3$. The 2-point bare truncated correlation function is diagonal in the color indices and is defined by 


$$
W^{(2)}(x, y)=Z^{(2)}(x, y)-\langle\phi(x)\rangle\left\langle\phi^{\dagger}(y)\right\rangle
$$

where

$$
Z^{(2)}(x, y)=\left\langle\phi(x) \phi^{\dagger}(y)\right\rangle
$$

The 2-point function $Z^{(2)}$ has the Fourier representation

$$
Z^{(2)}(x, y)=\int \frac{d^{d} p}{(2 \pi)^{d}} e^{i p \cdot(x-y)} \tilde{Z}^{(2)}(p)
$$

which satisfies the KL spectral representation [10]:

$$
\tilde{Z}^{(2)}(p)=c \delta^{d}(p)+\int_{0}^{\infty} d \mu \frac{\rho(\mu)}{p^{2}+\mu^{2}},
$$

where $\rho$ is the spectral density satisfying

$$
\int_{0}^{\infty} d \mu \rho(\mu)=1
$$

From Eq. (2.5) we obtain

$$
Z^{(2)}(x, y)=c+\int_{0}^{\infty} d \mu \rho(\mu) \frac{e^{-\mu|x-y|}}{4 \pi|x-y|}
$$

Let us put $y=0$ for convenience. Then, when the symmetry is broken, $W^{(2)}(x, 0) \rightarrow 0$ as $|x| \rightarrow \infty$. Therefore, from Eqs. (2.2) and (2.7) we obtain that $c=|\langle\phi(0)\rangle|^{2}$ which is different from zero if $T<T_{c}$, vanishing otherwise.

Using Eq. (2.6) it follows easily that the Fourier transform of the bare truncated 2-point correlation function satisfies the infrared bound [1], $\tilde{W}^{(2)}(p) \leq 1 / p^{2}$. Moreover, Griffiths correlation inequality [10 implies $\tilde{W}^{(2)}(p) \geq 0$. Therefore,

$$
0 \leq \tilde{W}^{(2)}(p) \leq \frac{1}{p^{2}} .
$$

The inequality (2.8) has an important consequence for the infrared behavior. At the CP, the bare correlation function behaves as $\tilde{W}^{(2)}(p) \sim 1 / p^{2-\eta}$ as $p \rightarrow 0$ and Eq. (2.8) implies therefore that $\eta \geq 0$. Note that in the above argument no reference is made to the global 
character of the symmetry group. Thus, we may think that the same rule should apply to the GL model where the gauge symmetry is local. We will see that this is not the case.

The bare Lagrangian of the GL model is

$$
L=\frac{1}{4} F^{2}+\left(D_{\mu} \phi\right)^{\dagger}\left(D_{\mu} \phi\right)+m^{2}|\phi|^{2}+\frac{u}{2}|\phi|^{4},
$$

where $F^{2}$ is a short for $F^{\mu \nu} F^{\mu \nu}, F^{\mu \nu}=\partial_{\mu} A_{\nu}-\partial_{\nu} A_{\mu}$, and $D_{\mu}=\partial_{\mu}+i e A_{\mu}$. At 1-loop, we obtain for $d=3, T \geq T_{c}$ and in the Coulomb gauge $\partial_{\mu} A_{\mu}=0$,

$$
\tilde{W}^{(2)}(p)=\frac{1}{p^{2}+m^{2}+\Sigma(p)},
$$

with the self-energy

$$
\Sigma(p)=-\frac{m}{2 \pi}\left(u+e^{2}\right)-\frac{e^{2}}{4 \pi|p|}\left(p^{2}-m^{2}\right)\left[\frac{\pi}{2}+\arctan \left(\frac{p^{2}-m^{2}}{2 m|p|}\right)\right] .
$$

In writing the above equations we have absorbed in the bare mass a contribution with a linear dependence on the ultraviolet cutoff $\Lambda$. Thus, $m^{2} \propto t$, where $t=\left(T-T_{c}\right) / T_{c}$ is the reduced temperature. The correlation function $\tilde{W}^{(2)}(p)$ at $p=0$ gives the susceptibility $\chi$. The divergence of the susceptibility at $T=T_{c}$ signals a phase transition. In terms of the correlation length $\xi=m_{r}^{-1}$, where $m_{r}$ is the renormalized mass, the susceptibility is written as $\chi=Z_{\phi} \xi^{2}$, where $Z_{\phi}$ is the wave-function renormalization. Here is the crucial point. For the $O(2)$ model, the 2-point correlation function diverges at $T_{c}$ only for $p=0$. The same is not true for the GL model. In fact, the above 1-loop calculation shows that for $|p|=p^{\prime}=e^{2} / 4$ the 2-point correlation function also diverges at $T_{c}$. Thus, we can define a second susceptibility $\chi^{\prime}=\tilde{W}^{(2)}\left(p^{\prime}\right)$. The existence of a second pole in $\tilde{W}^{(2)}$ implies that $Z_{\phi}>1$. Thus, the infrared bound Eq. (2.8) does not hold. If moreover we assume that the phase transition at $p=0$ is of second-order, we obtain that $\eta<0$. The same result holds at 2-loops and also in the $1 / N$ expansion. A negative value of $\eta$ is also found by means of non-perturbative RG [16] and in a recent Monte Carlo simulation [7]. This strange behavior needs an explanation and an interpretation. Note that not only the right hand side of (2.8) is violated but also its left hand side. The striking feature of this behavior is that $|\tilde{\phi}(p)|^{2} \geq 0$ 
for all $p$ but $\tilde{W}^{(2)}(p)=\left\langle|\tilde{\phi}(p)|^{2}\right\rangle<0$ if $0<|p|<p^{\prime}$. The average of the everywhere positive operator $|\tilde{\phi}(p)|^{2}$ is not positive everywhere! Thus, it seems that the corresponding effective Gaussian measure is not positive definite and, as a consequence, the functional integral is not well-defined. Of course, the KL representation cannot hold with a positive measure.

Let us explain the meaning of the susceptibility $\chi^{\prime}$. The fact that $\tilde{W}^{(2)}\left(p^{\prime}\right)$ diverges at $T_{c}$ means that a phase transition happens at finite distances. This is a typical feature of a first-order phase transition. The first- and second-order phase transition can be described at a same $T_{c}$ but at different momentum scales, $p=0$ for the second-order phase transition and $|p|=p^{\prime}$ for the first-order one. This shed new light in the RG fixed dimension approach at the CP of Refs. [18, 19], where two momentum scales are considered, defining in this way two characteristic lengths (note that for $T<T_{c}$ there are two lengths in the problem, namely, the correlation length $\xi$ and the penetration depth $\lambda$ ). For $T=T_{c}$, the fixed point structure is such that both phase transition regimes are contained in the RG flow diagram determined by dimensionless couplings $\hat{u}(\mu)=u_{r}(\mu) / \mu$ and $\hat{e}^{2}(\mu)=e_{r}^{2}(\mu) / \mu$, with $u_{r}$ and $e_{r}$ being the renormalized counterparts of $u$ and $e$. The regions of first- and second-order phase transition are separated by a line connecting the Gaussian fixed point and the so called tricritical fixed point [17]. This fixed point is infrared stable along the tricritical line and unstable in the the direction of $\hat{u}$. For momentum scales such that the couplings are at the left of the tricritical line, the phase transition is of first-order. Concerning the sign of $\eta$, it must be observed the following crossovers. The first one corresponds to zero charge, $\hat{e}^{2}=0$. In this case the flow is towards the $X Y_{3}$ fixed point and $\eta \geq 0$ ( $\eta=0$ at 1-loop). This situation is consistent with the infrared bound (2.8). The other crossover corresponds to the case where the couplings are over the tricritical line. In this situation the flow is towards the tricritical point. Both crossovers give a critical behavior consistent with a second-order like phase transition. It must be stressed, however, that the true second-order phase transition is governed by the infrared stable fixed point. The described crossovers are infrared stable only along the crossover lines, the tricritical line and the line $\hat{e}^{2}=0$. The critical regime 
associated to the tricritical line leads to $\eta<0$, in contrast to the $X Y_{3}$ crossover.

The singularity at $|p|=p^{\prime}$ can be interpreted in terms of the effective action. We will write the effective action in momentum space rather than in real space. Thus, if $\varphi$ and $a_{\mu}$ are the respective Legendre transformed fields of $\phi$ and $A_{\mu}$, we have $\Gamma=\int d^{3} p /(2 \pi)^{3} \tilde{\Gamma}(p)$, with

$$
\begin{aligned}
\tilde{\Gamma}(p) & =\frac{1}{2} \tilde{\Gamma}^{(2)}(p) \tilde{\varphi}_{i}(p) \tilde{\varphi}_{i}(-p)+\frac{1}{2} \tilde{\Gamma}_{\mu \nu}^{(2)}(p) \tilde{a}_{\mu}(p) \tilde{a}_{\nu}(-p) \\
& +\frac{1}{4} \int \frac{d^{3} q}{(2 \pi)^{3}} \int \frac{d^{3} k}{(2 \pi)^{3}} \tilde{\Gamma}^{(4)}(p, q, p-k, q+k)\left(\delta_{i j} \delta_{k l}+\delta_{i k} \delta_{j l}+\delta_{i l} \delta_{j k}\right) \tilde{\varphi}_{i}(p) \tilde{\varphi}_{j}(q) \tilde{\varphi}_{k}(p-k) \tilde{\varphi}_{l}(q+k) \\
& +\int \frac{d^{3} k}{(2 \pi)^{3}} \tilde{\Lambda}_{\mu}(p-k, p, k) \tilde{a}_{\mu}(p-k) \tilde{\varphi}_{1}(p) \tilde{\varphi}_{2}(k) \\
& +\frac{1}{2} \int \frac{d^{3} q}{(2 \pi)^{3}} \int \frac{d^{3} k}{(2 \pi)^{3}} \tilde{\Omega}(p, q, p-k, q+k) \tilde{\varphi}_{i}(p) \tilde{\varphi}_{i}(q) \tilde{a}_{\mu}(p-k) \tilde{a}_{\mu}(q+k)+(\text { h.o.t. }),
\end{aligned}
$$

where summation over repeated latin and greek indices is implied and we have written $\varphi=\left(\varphi_{1}+i \varphi_{2}\right) / \sqrt{2}$. Since $\tilde{\Gamma}^{(2)}(p)=1 / \tilde{W}^{(2)}(p)$, we have that at the CP the first term of the RHS of Eq. (2.12) vanishes when $|p|=p^{\prime}$ and is negative when $0<|p|<p^{\prime}$. Of course, when $\tilde{\Gamma}^{(2)}(p)$ is negative we must have a positive $\tilde{\Gamma}^{(4)}$ to ensure the stability of the effective action. In this paper we will not enter into the details of the stability conditions with respect to the 4-point function.

The physical picture that emerges from the behavior of the 2-point function is that of a tricritical Lifshitz point 35 38]. In fact, in scalar models for the Lifshitz points the 2-point function vanishes at the $\mathrm{CP}$ for a nonzero momentum value. In pure scalar models this can happen only if higher derivative Gaussian terms are present already at the tree level [35]. Remarkably, in the GL model the Lifshitz point is induced by the gauge field fluctuations [39]. The existence of a tricritical point in the GL model was established by Kleinert [5] using a disorder field theory obtained from duality arguments. In the disorder field theory scenario, an effective local scalar Lagrangian with disorder parameter $\psi$ is constructed. It has been shown that the effective quartic coupling in this model changes sign at some point in the coupling space of the original model. This characterizes an ordinary tricritical behavior in the disorder field theory. In the original GL model this tricritical point is of a Lifshitz type 
and that is the physical interpretation of the negative sign of $\eta$. Note that $\eta_{\text {dual }}$ is positive in the disorder field theory [9,21,23]. In scalar theories of the Lifshitz point the sign of $\eta$ is negative in dimensions $d_{c}-1$ where $d_{c}$ is the critical dimension of the model. For instance, a fixed dimension calculation in a $1 / N$ expansion gives for the isotropic Lifshitz point in $d=7\left(d_{c}=8\right.$ in this case) $\eta_{l 4} \approx-0.08 / N$ 《1].

A Lifshitz point behavior implies the existence of a modulated regime for the order parameter. This modulated regime should correspond to the type II regime and is analogous to the helical phase in scalar models of the Lifshitz point. The type I regime is analogous to the ferromagnetic or uniform order parameter phase in these models, the normal regime being the analog of the paramagnetic phase. The phase diagram should be therefore quite similar to the phase diagram of the R-S model [40]. The phase diagram of the R-S model is drawn in the $T-X$ plane where $X=S / R$ is the ratio between the couplings $S$ and $R$. In this phase diagram the line separating the helical phase from the ferromagnetic phase is a first order line. In the case of superconductors we should draw the phase diagram in a $T-\kappa^{2}$ plane, where $\kappa^{2}=u / 2 e^{2}$ is the square of the Ginzburg parameter. The phases paramagnetic, ferromagnetic and helical of the R-S model are replaced respectively by normal, type I and

type II. Experimentally, the modulated nature of the order parameter in the type II regime is seen upon applying an external magnetic field and corresponds to the Abrikosov vortex lattice 42 .

\section{WAVE FUNCTION RENORMALIZATION FROM FLUCTUATIONS AROUND THE HALPERIN-LUBENSKY-MA MEAN FIELD THEORY}

The HLM mean-field theory [12] neglects the order parameter fluctuations while including the gauge field fluctuations. For an uniform order parameter, the gauge field is integrated out exactly and a term proportional to $|\phi|^{3}$ with negative sign is generated in the free energy. The corresponding phase transition is found to be wekly first-order. RG calculations using the $\epsilon$-expansion confirms this scenario since no charged fixed point arises. A stable flow 
towards the infrared happens only at zero charge and the $X Y_{3}$ regime follows by taking $\epsilon=1$. The $X Y$ fixed point is unstable for arbitrarily small charge. This behavior remains even at 2-loop order 28]. Charged fixed points are obtained only by considering an order parameter with $N / 2$ complex components and in the limit of $N$ sufficiently large. Indeed, at 1-loop order charged fixed points are obtained if $N>365.9$. Interestingly, the critical value of $N$ decreases considerably already at 2-loops [28] and charged fixed points are found for $N>36$. More recently, by using Padé-Borel resummation of the $\epsilon$-expansion, Folk and Holovatch [15] succeeded in obtaining charged fixed points for the physical value $N=2$.

In this section we will evaluate the Gaussian fluctuations around the HLM mean-field theory. These fluctuations will not suffice for changing the order of the transition, and so it will remains first-order. Our interest here is the positivity properties of the 2-point correlation function in this fluctuation-corrected Gaussian approximation. This amounts in calculating the propagators associated to the HLM mean-field solution. Once this is done, the Gaussian measure necessary to compute the non-Gaussian fluctuations is determined. If this measure is not positive definite, then the functional integral is not well defined and all the theory is inconsistent. We will see that this is not the case.

Let us write $\phi=\left(\phi_{1}+i \phi_{2}\right) / \sqrt{2}$. By integrating out exactly the gauge field we obtain,

$$
Z=\lim _{a \rightarrow 0} \int D \phi_{1} D \phi_{2} \exp \left(-S_{e f f}\right)
$$

where the effective action

$$
\begin{aligned}
S_{e f f} & =\frac{1}{2} \operatorname{Tr} \ln \left[\hat{M}_{\mu \nu}(x-y ; a)\right] \\
& -\frac{e^{2}}{2} \int d^{3} x \int d^{3} y\left[\phi_{1}(x) \partial_{\mu}^{x} \phi_{2}(x)-\phi_{2}(x) \partial_{\mu}^{x} \phi_{1}(x)\right] \hat{D}_{\mu \nu}(x-y ; a)\left[\phi_{1}(y) \partial_{\nu}^{y} \phi_{2}(y)-\phi_{2}(y) \partial_{\nu}^{y} \phi_{1}(y)\right] \\
& +\int d^{3} x\left[\frac{1}{2} \phi_{1}\left(-\Delta+\delta m^{2}+m^{2}\right) \phi_{1}+\frac{1}{2} \phi_{2}\left(-\Delta+\delta m^{2}+m^{2}\right) \phi_{2}+\frac{u}{8}\left(\phi_{1}^{2}+\phi_{2}^{2}\right)^{2}\right]
\end{aligned}
$$

where we have introduced a mass counterterm $\delta m^{2}$, necessary to cancel tadpole divergences (see below). The operator $\hat{D}$ is the inverse of $\hat{M}$, the latter being given by

$$
\hat{M}_{\mu \nu}(x-y ; a)=\delta^{3}(x-y)\left\{\left[-\Delta+e^{2}\left(\phi_{1}^{2}+\phi_{2}^{2}\right)\right] \delta_{\mu \nu}+(1-1 / a) \partial_{\mu} \partial_{\nu}\right\}
$$


where $a$ is the gauge fixing parameter. In Eq. (3.1), the limit $a \rightarrow 0$ is taken in order to inforce the Coulomb gauge condition. Now, we consider small fluctuations around $\phi_{i}=v \delta_{i 1}$, where $v=$ const is the solution of

$$
\frac{\delta S_{e f f}}{\delta \phi_{i}}=0
$$

In this case it is legitimate to truncate $S_{\text {eff }}$ up to quadratic order in the fluctuating fields $\delta \phi_{1}$ and $\delta \phi_{2}$. The result is

$$
\begin{aligned}
S_{e f f} & =S_{e f f}^{H L M} \\
& +\frac{1}{2} \int d^{3} x \int d^{3} y\left\{\delta \phi _ { 1 } ( x ) \left[\left(-\Delta+\delta m^{2}+3 \bar{m}^{2}+m^{2}+e^{2} D_{\mu \mu}(0)\right) \delta^{3}(x-y)\right.\right. \\
& \left.-2 e^{2} M^{2} D_{\mu \nu}(x-y) D_{\nu \mu}(y-x)\right] \delta \phi_{1}(y) \\
& \left.+\delta \phi_{2}(x)\left[\left(-\Delta+\delta m^{2}+\bar{m}^{2}++m^{2}+e^{2} D_{\mu \mu}(0)\right) \delta^{3}(x-y)-M^{2} \partial_{\mu}^{x} \partial_{\nu}^{y} D_{\mu \nu}(x-y)\right] \delta \phi_{2}(y)\right\},
\end{aligned}
$$

where $\bar{m}^{2}=u v^{2} / 2, M^{2}=e^{2} v^{2}$ and $S_{e f f}^{H L M}$ corresponds to the HLM mean-field free energy [12]. Also,

$$
D_{\mu \nu}(x-y)=\int \frac{d^{3} p}{(2 \pi)^{3}} e^{i p \cdot(x-y)} \tilde{D}_{\mu \nu}(p)
$$

is the operator $\hat{D}$ for $\delta \phi_{1}=\delta \phi_{2}=0$. In the Coulomb gauge,

$$
\tilde{D}_{\mu \nu}(p)=\frac{1}{p^{2}+M^{2}}\left(\delta_{\mu \nu}-\frac{p_{\mu} p_{\nu}}{p^{2}}\right)
$$

which implies

$$
\int d^{3} x \int d^{3} y \partial_{\mu}^{x} \partial_{\nu}^{y} D_{\mu \nu}(x-y) \delta \phi_{2}(x) \delta \phi_{2}(y)=0
$$

Now we see that the counterterm $\delta m^{2}$ is necessary in order to cancel the linear cutoff dependence coming from the tadpole term $e^{2} D_{\mu \mu}(0)$. Therefore, the $\delta \phi_{1}$ propagator is

$$
G_{11}(p)=\frac{1}{p^{2}+m^{2}+3 \bar{m}^{2}+\Sigma_{11}(p)},
$$

where the self-energy $\Sigma_{11}(p)$ is given by

$$
\Sigma_{11}(p)=e^{2} D_{\mu \mu}(0)-2 e^{2} M^{2} \int \frac{d^{3} k}{(2 \pi)^{3}} \tilde{D}_{\mu \nu}(k-p) \tilde{D}_{\nu \mu}(k) .
$$


By evaluating the integrals in Eq. (3.10) we obtain

$$
\begin{aligned}
\Sigma_{11}(p) & =-\frac{e^{2} M}{2 \pi}-e^{2}\left[\frac{M}{4 \pi}-\frac{p}{8}-\frac{M^{2}}{16 p}+\frac{p^{4}+8 M^{4}+4 M^{2} p^{2}}{8 \pi p M^{2}} \arctan \left(\frac{p}{2 M}\right)\right. \\
& \left.-\frac{\left(p^{2}+M^{2}\right)^{2}}{8 \pi p M^{2}} \arctan \left(\frac{p^{2}-M^{2}}{2 p M}\right)\right] .
\end{aligned}
$$

The $\delta \phi_{2}$ propagator is given simply by

$$
G_{22}(p)=\frac{1}{p^{2}+m^{2}+\bar{m}^{2}-\frac{e^{2} M}{2 \pi}} .
$$

Note that the above calculation differs from the usual 1-loop result. In an ordinary 1-loop calculation we integrate out the quadratic fluctuations around the solution $v=\left(-2 m^{2} / u\right)^{1 / 2}$ corresponding to the tree-level with $\mathbf{A}=0$. Above, we integrated out $\mathbf{A}$ first and then we computed the Gaussian fluctuations around a solution $v$ given by Eq. (3.4), which already contain magnetic fluctuations. Eq. (3.4) have the following solutions:

$$
\begin{gathered}
v=0 \\
|v|=\frac{e^{3}}{2 \pi u} \pm \frac{1}{u} \sqrt{\frac{e^{6}}{4 \pi^{2}}-2 u m^{2}}
\end{gathered}
$$

Thus, in the ordered phase given by Eq. (3.14) we have $m^{2}+\bar{m}^{2}-e^{2} M / 2 \pi=0$ and therefore the $\delta \phi_{2}$ propagator $G_{2} 2(p)$ is massless. Then, the fluctuating field $\delta \phi_{2}$ is the would-be Goldstone boson of the theory. When $m^{2}=e^{6} /\left(8 \pi^{2} u\right)$ the square root in Eq. (3.14) vanishes. This value of $m^{2}$ corresponds to a point of non-analyticity of $v$ as a function of $m^{2}$. Indeed, the derivative of $v$ with respect to $m^{2}$ diverges for $m^{2}=e^{6} /\left(8 \pi^{2} u\right)$. Thus, if we expand the denominator of $G_{11}$ for $p$ small we obtain,

$$
G_{11}(p)=\frac{1}{\left(1+\frac{5}{24 \pi} \frac{e^{2}}{M}\right) p^{2}+m^{2}+3 \bar{m}^{2}-\frac{e^{2} M}{\pi}+O\left(p^{4}\right)}
$$

and we see that the susceptibilities $\chi_{i}=G_{i i}(0)(i=1,2)$ diverge together if $m^{2}=e^{6} /\left(8 \pi^{2} u\right)$. This singular behavior of the susceptibilities is not associated to any phase transition. It is just an artifact of our fluctuation-corrected Gaussian approximation. The singularity of $\chi_{i}$ for $m^{2}=e^{6} /\left(8 \pi^{2} u\right)$ is inherited from the non-analytic behavior of $v$ for this value of 
$m^{2}$. Once the non-Gaussian fluctuations are taken into account and a full renormalization of mass and coupling constants is done, this artifact disappears. On the other hand, if $m^{2}<e^{6} /\left(8 \pi^{2} u\right), \chi_{2}$ diverge but not $\chi_{1}$. In this fluctuation induced phase transition scenario $v=\langle\phi\rangle$ is different from zero at the CP, a typical behavior of a first-order transition, as we have already discussed in section II. Note that in this calculation the correlation functions diverge only at $p=0$. As a consequence, the wave function renormalizations $Z_{i} \leq 1$. By putting $v=e^{3} /(\pi u)$ which corresponds to $m^{2}=0$ in Eq. (3.15), we obtain

$$
Z_{1}=\frac{1}{1+\frac{5}{12} \kappa^{2}}<1
$$

Therefore, the corresponding Gaussian measure is positive definite and the non-Gaussian fluctuations can be calculated by means of this measure. The non-Gaussian fluctuations will ultimately make $Z_{1}>1$, violating again the KL representation.

\section{THE VECTOR POTENTIAL ANOMALOUS DIMENSION AND ITS CONSEQUENCES FOR THE CRITICAL DYNAMICS IN SUPERCONDUCTORS}

One important feature of the $I X Y_{3}$ universality class is the scaling $\lambda \sim \xi[18,21,30,23$, where $\lambda$ and $\xi$ are the penetration depth and correlation length, respectively. This scaling contrast with the $X Y_{3}$ behavior, where $\lambda \sim \xi^{1 / 2}$ [1],31]. The reason for this different behavior comes from the magnetic fluctuations, which in the $X Y_{3}$ universality class play no role. In the $X Y_{3}$ regime the magnetic vector potential has no anomalous dimension. Concerning the scaling of the penetration depth, it was argued in Refs. [18,23 that the vector potential anomalous dimension contributes in such a way that we have in general $\lambda \sim \xi^{\left(\eta_{A}+d-2\right) / 2}$. Thus, when the magnetic fluctuations are negligeable we have $\eta_{A}=0$ and $\lambda \sim \xi^{(d-2) / 2}$, implying in this way a penetration depth exponent $\nu^{\prime}=\nu(d-2) / 2$ with $\nu \approx 2 / 3$ when $d=3$. On the other hand, if we take into account the magnetic fluctuations, we have that $\eta_{A}=4-d$ and $\lambda \sim \xi$ implying $\nu^{\prime}=\nu$. The critical exponent $\nu$ is the same in both $X Y_{3}$ and $I X Y_{3}$ universality classes [9,21, 19, 23, 22, 30] and we obtain that $\nu^{\prime} \approx 1 / 3$ and $\nu^{\prime} \approx 2 / 3$ 
for the $X Y_{3}$ and $I X Y_{3}$ regimes, respectively. Note that only the thermodynamic exponents coincide in these two $X Y$ regimes. As we have already seen, the anomalous dimensions are not the same.

The frequency-dependent conductivity $\sigma(\omega)$ scales differently in a magnetic fluctuation regime. For $T<T_{c}$ we have that $\sigma(\omega) \sim e^{2} \rho_{s} /(-i \omega)$, where $\rho_{s}$ is the superfluid density. Near a charged fixed point we have $e^{2} \sim \xi^{-\eta_{A}}$ and therefore,

$$
\sigma(\omega) \sim \xi^{2-d+z-\eta_{A}},
$$

where $z$ is the dynamical exponent and we have used the Josephson relation $\rho_{s} \sim \xi^{2-d}$ [44, 45, 23]. Again, by neglecting the magnetic fluctuations we recover the usual scaling [31]. The $X Y$ scaling proposed by Fisher et al. [31] was verified recently by Wickham and Dorsey [32], who calculated $\sigma(\omega)$ using the Kubo formula to $O\left(\epsilon^{2}\right)$ in the $\epsilon=4-d$-expansion.

Since in the magnetic fluctuation regime $\eta_{A}=4-d$, we obtain

$$
\sigma(\omega) \sim \xi^{z-2},
$$

a result independent of the dimension. This independence of the dimension in the scaling behavior (4.2) seems to be a special feature of the charged fixed point. Note that already in the case of the penetration depth we have obtained $\nu^{\prime}=\nu$ instead of the dimension dependent result $\nu^{\prime}=\nu(d-2) / 2$ of the $X Y$ regime. The scaling given in Eq. (4.2) has been obtained before by Mou [33] who used a completely different argument. Our argument is much more simple and follows from the exact value of the vector potential anomalous dimension. However, the dynamical exponent $z$ is not be the same as in the uncharged model, as was claimed in Ref. [33]. The Monte Carlo simulations of Lidmar et al. [50] show very clearly that this is not the case and that the value of $z$ is enhanced by magnetic fluctuations. However, Lidmar et al. fitted their Monte Carlo data to $\sigma \sim|t|^{\nu(d-2-z)}$ instead of using the scaling (4.2). Since $\eta_{A}=1$ in $d=3$, we conclude that the numerical result of Ref. [50] should be shifted to obtain $z \approx 3.7$ instead of $z \approx 2.7$. This surprisingly high value of $z$ could be, however, a matter of controversy. It may be a consequence of the way the 
authors of Ref. [50] modelled the $I-V$ characteristics of the $I X Y_{3}$ regime. For instance, Ampère's law is neglected in their approach.

From the experimental side, the work of Booth et al. [51] fit reasonably the value $z=2.7$ but they assume also a scaling with $\eta_{A}=0$. Anyway, in the case of Ref. [51] it is more probable that the critical region probed does not correspond to a $I X Y_{3}$ universality class. In this case $\eta_{A}=0$ would be a legitimate assumption.

The scaling Eq. (4.2) is also relevant in other situations. For example, in the Bose-glass transition the conductivity perpendicular to the columnar defects is argued to obey a scaling exactly as in Eq. (4.2) 27,26]. Although Eq. (4.2) is a zero field scaling, it should apply in the nonzero field situation of the Bose-glass transition in the direction perpendicular to the columnar defects but not in the longitudinal direction. The reason for this behavior comes from the fact that in the perpendicular direction a transverse Meissner effect happens, implying in this way a zero field like situation. The Bose-glass transition is an example which shows that at high fields the magnetic thermal fluctuations may be experimentally important and observable.

\section{CONCLUSION}

In this paper we have discussed some new features of the superconducting phase transition. The important role of the anomalous dimensions has been emphasized. However, the critical behavior disussed in this paper is relevant only near a charged fluctuation critical regime. The relevance of the ideas discussed here to high-temperature superconductors (HTSCs) may be questioned. Usually the HTSCs have very high values of $\kappa$, typically in the range $70-100$. For this reason, it is generally assumed that magnetic fluctuations do not play an important role. This is in fact the case in the extreme type II limit, that is, $\kappa \rightarrow \infty$. For extreme type II superconductors, the local magnetic induction equals the applied magnetic field and the constraint $\nabla \times \mathbf{A}=\mathbf{H}$ applies 22. In the presently accessible

critical region, the HTSCs seem to be well approximated by an extreme type II limit. In 
this case the $X Y_{3}$ regime dominate at zero or low magnetic fields. The $X Y_{3}$ behavior has been probed with considerable confidence in $\mathrm{YBa}_{2} \mathrm{Cu}_{3} \mathrm{O}_{7-\delta}$ (YBCO) crystal samples [2, 3. 46]. For $\mathrm{Bi}_{2} \mathrm{Sr}_{2} \mathrm{CaCu}_{2} \mathrm{O}_{8+\delta}$ (BSCCO), however, the situation is less clear due to the experimental difficulties involved. Specific heat measurements seem to indicate that the universality class is not $X Y_{3}$ [47]. The apparent failure of the $X Y_{3}$ scaling in BSCCO seems also to be corroborated by the penetration depth data [48]. However, inhomegeneities and finite size effects can play a significant role in BSCCO and it may happen that it obeys also a $X Y_{3}$ scaling [49].

The $I X Y_{3}$ behavior, on the other hand, seems to be not presently accessible. In fact, penetration depth data from YBCO fulfill very well the scaling relation $\nu^{\prime}=\nu / 2$ [3], agreeing with the $X Y_{3}$ behavior. Thus, in order to check the theoretical predictions concerning the $I X Y_{3}$ regime, we have to compare these mainly to Monte Carlo simulations. For instance, the scaling relation $\nu^{\prime}=\nu$ with $\nu \approx 2 / 3$ was well verified by Olsson and Teitel [30]. The value $\eta \approx-0.18$ was obtained by Nguyen and Sudbø [7]. The dynamical exponent $z$ was studied by Lidmar et al. [50] both in the $X Y_{3}$ and in the $I X Y_{3}$ regimes. However, as discussed in section IV, they assumed the same scaling for the frequency dependent conductivity in both regimes. This does not invalidate their data, which remain useful and lead to the prediction $z \approx 3.7$ instead of $z \approx 2.7$. While presently there is little hope in checking these predictions in zero field experiments, further Monte Carlo simulations can be done in order to obtain a definitive answer. As far as real experiments are concerned, we have pointed out that the scaling given in Eq. (4.2) holds for the condcutivity perpendicular to the columnar defects in a Bose-glass transition [26,27]. Unfortunately, in this nonzero field regime we are unable to estimate the value of $z$ with the arguments presented in this paper. It is worth to mention, however, that an experimental value $z \approx 5.3$ was probed recently by Klein et al. [52] for the Bose-Glass transition in the fully isotropic compound $(\mathrm{K}, \mathrm{Ba}) \mathrm{BiO}_{3}$ with columnar defects. 


\section{ACKNOWLEDGMENTS}

The author would like to acknowledge A. Sudbø for sending the paper Ref. [8] prior to publication. Much of the present work originated from discussions with A. Sudbø and Z. Tešanović and the author is indebted to them. The author acknowledges also C. de Calan for numerous discussions. 


\section{REFERENCES}

[1] C. J. Lobb, Phys. Rev. B 36, 3930 (1987).

[2] M. B. Salamon, J. Shi, N. Overend and M. A. Howson, Phys. Rev. B 47, 5520 (1993); N. Overend, M. A. Howson and I. D. Lawrie, Phys. Rev. Lett. 72, 3238 (1994).

[3] S. Kamal, D. A. Bonn, N. Goldenfeld, P. J. Hirschfeld, R. Liang and W. N. Hardy, Phys. Rev. Lett. 73, 1845 (1994); S. Kamal, R. Liang, A. Hosseini, D. A. Bonn and W. N. Hardy, Phys. Rev. B 58, R8933 (1998).

[4] C. Dasgupta and B. I. Halperin, Phys. Rev. Lett. 47, 1556 (1981).

[5] H. Kleinert, Lett. Nuovo Cimento 35, 405 (1982); see also the textbook, H. Kleinert, Gauge Fields in Condensed Matter, vol. 1, World Scientific, 1989.

[6] A. Sudbø, A. K. Nguyen and J. Hove, cond-mat/9907386, to appear in Physica B.

[7] A. K. Nguyen and A. Sudbø, Phys. Rev. B 60, 15307 (1999).

[8] J. Hove and A. Sudbø, Phys. Rev. Lett 84, 3426 (2000).

[9] M. Kiometzis, H. Kleinert and A. M. J. Schakel, Phys. Rev. Lett. 73, 1975 (1994).

[10] J. Glimm and A. Jaffee, Quantum Physics - A Functional Integral Point of View, 2nd edition (Springer-Verlag, Berlin, 1987).

[11] J. Fröhlich, B. Simon and T. Spencer, Commun. Math. Phys. 50, 79 (1976).

[12] B. I. Halperin, T. C. Lubensky and S.-K. Ma, Phys. Rev. Lett. 32, 292 (1974); J.-H. Chen, T. C. Lubensky and D. R. Nelson, Phys. Rev. B 17, 4274 (1978).

[13] S. Hikami, Prog. Theor. Phys. 62, 226 (1979); I. D. Lawrie, Nucl. Phys. B 200 [FS 14] $1(1982)$.

[14] L. Radzihovsky, Europhys. Lett. 29, 227 (1995).

[15] R. Folk and Y. Holovatch, J. Phys. A 29, 3409 (1996). 
[16] B. Bergerhoff, F. Freire, D. F. Litim, S. Lola and C. Wetterich, Phys. Rev. B 53, 5734 (1996).

[17] The existence of a tricritical point was predicted by H. Kleinert; see Ref. [5].

[18] I. F. Herbut and Z. Tešanović, Phys. Rev. Lett. 76, 4588 (1996); I. D. Lawrie, ibid., 78, 979 (1997); I. F. Herbut and Z. Tešanović, ibid. 78, 980 (1997).

[19] C. de Calan, A. P. C. Malbouisson, F. S. Nogueira and N. F. Svaiter, Phys. Rev. B 59, 554 (1999).

[20] F. S. Nogueira, Europhys. Lett. 45, 612 (1999).

[21] I. F. Herbut, J. Phys. A 30, 423 (1997).

[22] Z. Tešanović, Phys. Rev. B 59, 6449 (1999).

[23] C. de Calan and F. S. Nogueira, Phys. Rev. B 60, 4255 (1999).

[24] The value of $\eta$ given in Ref. [18] matchs with the one obtained in Ref. [7]. Almost all calculations give values around $-0.10:-0.10$ in Ref. [15], -0.15 in Ref. [16] and -0.14 in Ref. [19]. The most negative value is obtained in Ref. [14], $\eta=-0.38$.

[25] M. Kiometzis and A. M. J. Schakel, Int. J. Mod. Phys. B 7, 4271 (1993).

[26] D. R. Nelson and V. M. Vinokur, Phys. Rev. B 48, 13060 (1993); ibid. 61, 5917 (2000).

[27] J. Lidmar and M. Wallin, Europhys. Lett. 47, 494 (1999).

[28] S. Kolnberger and R. Folk, Phys. Rev. B 41, 4083 (1990); The earliest two-loop calculation of the renormalization group functions of the GL model was done in an unpublished Master's thesis by a student of Prof. Kleinert, J. Tessmann, in 1984.

[29] J. Zinn-Justin, Quantum Field Theory and Critical Phenomena, 2nd edition (Oxford, 1993).

[30] P. Olsson and S. Teitel, Phys. Rev. Lett. 80, 1964 (1998). 
[31] D. S. Fisher, M. P. A. Fisher and D. A. Huse, Phys. Rev. B 43, 130 (1991).

[32] R. A. Wickham and A. T. Dorsey, Phys. Rev. B 61, 6945 (2000).

[33] C.-Y. Mou, Phys. Rev. B 55, R3378 (1997).

[34] M. Kiometzis, H. Kleinert and A. M. J. Schakel, Fortschr. Phys. 43, 697 (1995) and references therein.

[35] R. M. Hornreich, M. Luban and S. Shtrikman, Phys. Rev. Lett. 35, 1678 (1975); R. M. Hornheich, J. Magn. Magn. Mater. 15-18, 387 (1980).

[36] W. Selke, in Phase Transitions and Critical Phenomena, edited by C. Domb and J. L. Lebowitz (Academic Press, London, 1992), Vol. 15, pages 1-72.

[37] I. Nasser and R. Folk, Phys. Rev. B 52, 15799 (1995); I. Nasser, A. Abdel-Hady and R. Folk, Phys. Rev. B 56, 154 (1997).

[38] C. Mergulhão Jr. and C. E. I. Carneiro, Phys. Rev. B 59, 13954 (1999).

[39] Recently, a Lifshitz point induced by fluctuations was found in a non-commutative scalar theory [S. S. Gubser and S. L. Sondhi, hep-th/0006119 (unpublished)]. For this class of scalar fields the kinetic term does not contains higher order derivatives and is the non-commutative character of the interaction that induces the Lifshitz point.

[40] S. Redner and H. E. Stanley, Phys. Rev. B 16, 4901 (1977); J. Phys. C 10, 4765 (1977).

[41] R. M. Hornreich, M. Luban and S. Shtrikman, Phys. Lett. A 55, 269 (1975).

[42] A. A. Abrikosov, Soviet Phys. (JETP) 5, 1174 (1957).

[43] By minimal subtraction we do not mean necessarily dimensional regularization. Minimal subtraction can be done also in fixed dimension. See R. Schloms and V. Dohm, Nucl. Phys. B 328, 639 (1989).

[44] B. D. Josephson, Phys. Lett. 21, 608 (1966). 
[45] M. E. Fisher, M. N. Barber and D. Jasnow, Phys. Rev. A 8, 1111 (1973).

[46] A. Junod, M. Roulin, B. Revaz and A. Erb, to appear in the proceedings of the LT22 to be published in Physica B, and references therein.

[47] A. Junod, A. Erb and C. Renner, Physica C 317-318, 333 (1999).

[48] T. Jacobs, S. Sridhar, Q. Li, G. D. Gu and N. Koshizuka, Phys. Rev. Lett. 75, 4516 (1995).

[49] T. Schneider, Private Communication.

[50] J. Lidmar, M. Wallin, C. Wengel, S. M. Girvin and A. P. Young, Phys. Rev. B 58, 2827 (1998).

[51] J. C. Booth et al., Phys. Rev. Lett. 77, 4438 (1996).

[52] T. Klein, A. Conde-Gallardo, I. Joumard, J. Marcus, C. J. van der Beek and M. Konczykowski, Phys. Rev. B 61, R3830 (2000). 